\title{
Hot Spot Emission from a Freely Precessing Neutron Star
}

\section{Citation}

Heyl, Jeremy S., and Lars Hernquist. 2002. "Hot Spot Emission from a Freely Precessing Neutron Star." The Astrophysical Journal 567 (1): 510-14. https://doi.org/10.1086/338547.

\section{Permanent link}

http://nrs.harvard.edu/urn-3:HUL.InstRepos:41381848

\section{Terms of Use}

This article was downloaded from Harvard University's DASH repository, and is made available under the terms and conditions applicable to Other Posted Material, as set forth at http:// nrs.harvard.edu/urn-3:HUL.InstRepos:dash.current.terms-of-use\#LAA

\section{Share Your Story}

The Harvard community has made this article openly available.

Please share how this access benefits you. Submit a story.

\section{Accessibility}




\title{
Hotspot Emission from a Freely Precessing Neutron Star
}

\author{
Jeremy S. Heyl \\ Theoretical Astrophysics, mail code 130-33, California Institute of Technology, Pasadena CA \\ 91125 \\ and \\ Lars Hernquist \\ Harvard-Smithsonian Center for Astrophysics, 60 Garden Street Cambridge, MA 02138
}

\begin{abstract}
Recent observations of $1 \mathrm{E}$ 161348-5055 the neutron-star candidate at the center of the supernova remnant RCW 103, show that a component of its emission varies sinusoidally with a period of approximately six hours. We argue that this period is what one would expect for a freely precessing neutron star with a spin period of about one second. We produce light curves for a freely precessing neutron star with a hotspot. By a suitable choice of parameters, we obtain light curves which are constant with rotational phase when the flux from the star reaches a maximum. At other phases of the precession, the flux varies as the star rotates but the total flux decreases by a factor of several. These models can explain the behavior observed from 1E 161348-5055 and predict that the spin period should be detectable at minimum flux from sufficiently sensitive measurements.
\end{abstract}

\section{Introduction}

Garmire et al. (2000) have noted that the x-ray source, 1E 161348-5055, located near the center of the supernova remnant RCW 103, has a sinusoidal light curve with a period of approximately six hours. The authors suggest that this period implies that the x-ray source has a low-mass companion with a six hour orbital period. In this paper, we examine an alternative possibility, that $1 \mathrm{E}$ 161348-5055 is a freely precessing neutron star with a precession period of about six hours.

The varying spectrum from 1E 161348-5055 is similar to a blackbody yielding an effective area of a small fraction of a square kilometer (Garmire, private communication), much less than the surface area of a neutron star; therefore, the situation could be well approximated by a point source (a hotspot) on the surface of a freely-precessing neutron star.

Free precession has often been invoked to explain long-period variability in neutron stars and neutron-star systems. Brecher (1975) attributed the 35-day cycle of Her X-1 (Tananbaum et al. 1972) to the free precession of the neutron star secondary. Jones (1988) explained timing 
residuals in the Crab pulsar as arising from small amplitude free precession of the neutron star. Cadez \& Galicic (1996) found a small amplitude modulation in the optical flux from the Crab pulsar which Cadez, Galicic \& Calvani (1997) cite as evidence of free precession.

In $\delta 2$ we outline the kinematics of free precession, and in $\S 3$, we review the equations that determine the trajectory of light leaving the surface of the neutron star. 任 presents the light curves as a function of precessional and rotational phase for Newtonian, relativistic and ultracompact neutron stars with a emission from a hotspot. Finally, $\$ 5$ places the results in a greater context.

\section{Free Precession}

A body will precess freely if its rotation axis does not coincide with one of its principal axes. More specifically, if the body is only slightly prolate or oblate, the angular velocity vector $(\vec{\omega})$ of the star will make a constant angle $(\kappa)$ with one of the principal axes (the 3 -axis), forming the body cone, and will trace a cone in space (the space cone) with half-opening angle $\kappa$ as well. The rate of the precession is given by (Goldstein 1980)

$$
\Omega=\frac{I_{3}-I_{1}}{I_{1}} \omega_{3}=\epsilon \omega_{3} .
$$

Values of $\epsilon=10^{-3}-10^{-4}$ agree with the glitching behavior of neutron stars and the inferred

gravitational-radiation spindown from the Crab pulsar (Shapiro \& Teukolsky 1983). Forced precession by an orbiting companion typically has a frequency lower by a factor of $2 /\left(3 \Omega_{*} \omega_{3}\right)$ where $\Omega_{*}$ is the angular frequency of the orbit.

Internal magnetic fields may distort a neutron star significantly. Ostriker \& Gunn (1969) estimate the distortion of a neutron star due to internal fields,

$$
\epsilon \simeq 4 \times 10^{-6}\left(3\left\langle B_{p, 15}^{2}\right\rangle-\left\langle B_{\phi, 15}^{2}\right\rangle\right)
$$

where $B_{15}$ is the value of the magnetic field in units of $10^{15} \mathrm{G}$ and $\langle\ldots\rangle$ denotes a volume-weighted average over the star. Here, $B_{p}$ and $B_{\phi}$ refer to the poloidal and azimuthal components of the magnetic field, respectively. One expects the internal fields of the neutron star to be significantly larger than the field inferred by magnetic dipole radiation due to the contribution of higher multipoles and the concentration of magnetic flux in flux tubes (e.g. Pines \& Alpar 1985).

We would like to calculate the light curve from a hotspot on the surface of the freely precessing neutron star. Let us take the center of the cone that the angular velocity moves along to be the $\overrightarrow{\mathbf{z}}$-axis. Our line of sight $(\overrightarrow{\mathbf{O}})$ makes an angle $\xi$ with this axis and forms a plane with $\overrightarrow{\mathbf{z}}$-axis. We measure the phase of the precession $(\phi)$ relative to intersection of the space cone with this plane.

The plane containing $\overrightarrow{\mathbf{z}}$ and $\overrightarrow{\mathbf{O}}$ also intersects the body cone. The angular momentum of the star points along the $\overrightarrow{\mathbf{z}}$-axis. In the body frame, the angular velocity of the star traces a cone centered on a principal axis of the star. We call this principal axis $\overrightarrow{\mathbf{3}}$. The hotspot is located at $\vec{\mu}$ 
which makes an angle $\beta$ with $\overrightarrow{\mathbf{3}}$. Let us freeze the precession and the rotation of the star when $\overrightarrow{\mathbf{3}}$ points along $\overrightarrow{\mathbf{z}}$ (see Figure $\mathbb{1}$ ). At this orientation, the angle between the $\overrightarrow{\mathbf{3}}-\overrightarrow{\mathbf{O}}$ plane and the $\overrightarrow{\mathbf{3}}-\vec{\mu}$ plane is $\gamma$.

Using spherical trigonometry (see Figure 1) yields the angle between the line of sight and the rotation axis, $\zeta(\phi)$, and the angle between the hotspot and the rotation axis $\alpha(\phi)$.

$$
\begin{aligned}
\cos \zeta(\phi) & =\cos \kappa \cos \xi+\sin \kappa \sin \xi \cos \phi \\
\cos \alpha(\phi) & =\cos \kappa \cos \beta+\sin \kappa \sin \beta \cos (\phi-\gamma)
\end{aligned}
$$

The star rotates as well as precesses. The phase of the rotation is given by $\eta$. When $\eta=0$ the hotspot lies in the $\vec{\omega}-\overrightarrow{\mathbf{O}}$ plane. The angle between the line of sight and the hotspot is $\theta$ and is given by

$$
\cos \theta=\cos \alpha(\phi) \cos \zeta(\phi)+\sin \alpha(\phi) \sin \zeta(\phi) \cos \eta
$$

If the hotspot emits isotropically, and we neglect gravitational lensing, the observed flux from the hotspot is simply proportional to $\cos \theta$ for $|\theta|<\pi / 2$ and zero otherwise.

\section{Gravitational Lensing}

Page (1995) presents a detailed treatment of the gravitational lensing of the surface of a neutron star. Since the light trajectory is bent, the zenith angle of our detector $(\delta)$ as seen from the hotspot is no longer equal to $\theta$. They are related by

$$
\theta(x)=\int_{0}^{y} \frac{x d u}{\sqrt{(1-2 y) y-(1-2 u) u^{2} x^{2}}}
$$

where $y=G M / R c^{2}$ and $x=\sin \delta$. For $y<1 / 3$, the image of the hotspot $i$ will be visible if $\theta_{i}+2 \pi j<\theta(1)$. The flux from the hotspot is proportional to

$$
\left.\sum_{j} \frac{x\left(\theta_{i}+2 \pi j\right)}{\sin \left(\theta_{i}+2 \pi j\right)} \frac{\mathrm{d} x}{\mathrm{~d} \theta}\right|_{\theta=\theta_{i}+2 \pi j}
$$

such that $\left|\theta_{i}+2 \pi j\right| \leq \theta(1)$.

For $y>1 / 3$ the surface of the neutron star lies below the circular photon orbit; therefore, each hotspot yields an infinite number of images (Shapiro \& Teukolsky 1983):

$$
\lim _{x \rightarrow x_{\max }} \theta(x ; y>1 / 3)=\infty .
$$

where

$$
x_{\max }=3 \sqrt{3} y \sqrt{1-2 y} .
$$




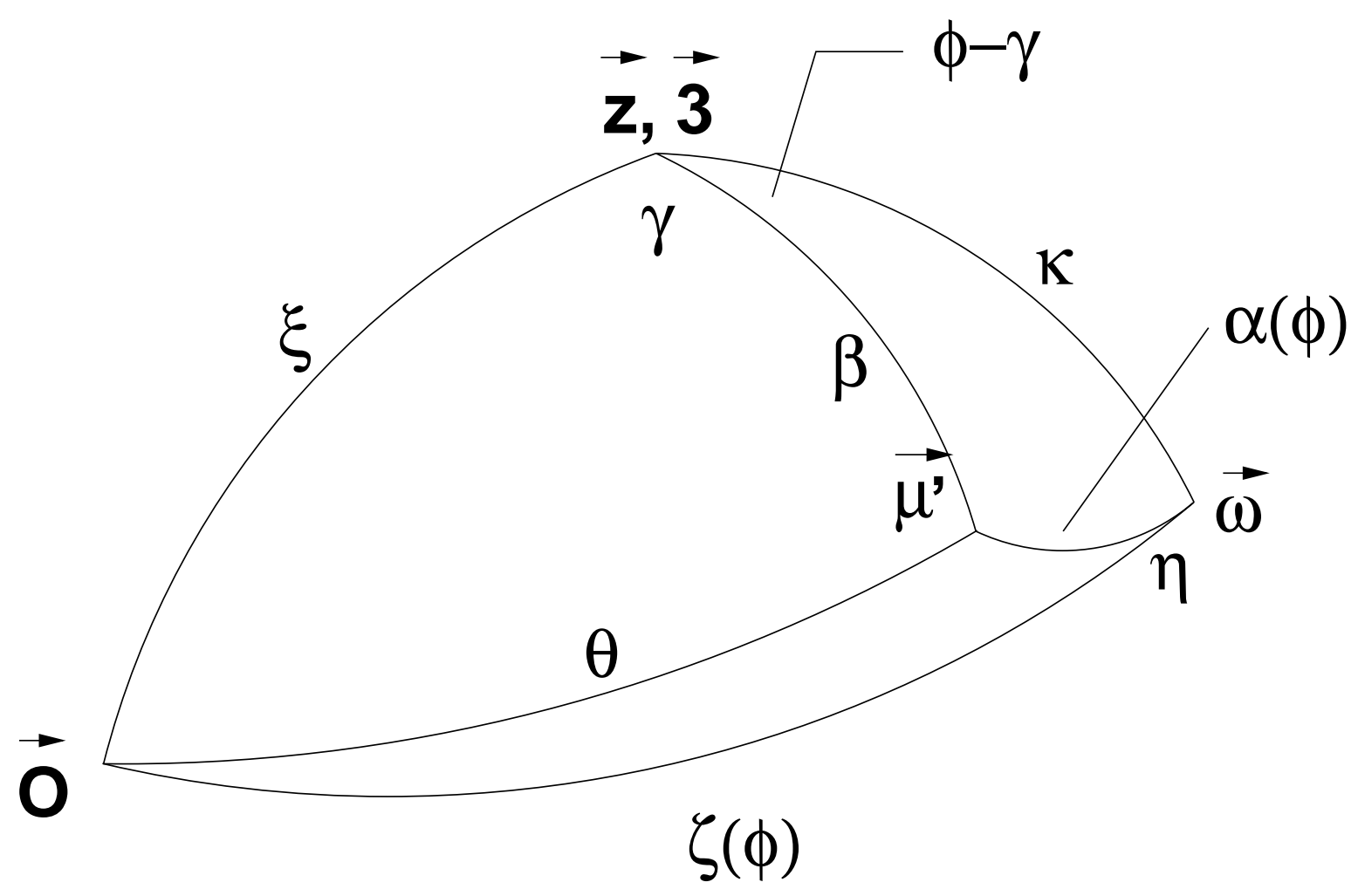

Fig. 1.- Definitions of various angles 


\section{Light Curves}

To construct a light curve, we must specify several angles $(\xi, \kappa, \beta$ and $\gamma)$. During some portion of the precession period, the observed flux will be constant with orbital phase, if either $\beta=\kappa$ or $\xi=\kappa$. In the first case, the angular velocity vector will coincide with the location of the hotspot on the star. In the second case, the angular velocity vector will point along the line of sight once during each precession.

\section{1. $\beta=\kappa=90^{\circ}$}

To maximize the flux during the portion of the precessional period where the flux does not vary with rotational phase, we take $\gamma=0$ and $\beta=\kappa=90^{\circ}$, and to minimize the flux during the rest of the precession, we take $\xi=\theta(1)$. If $\gamma \neq 0$, the maximum flux will not occur during that portion of precession when the flux does not vary with rotational phase. Taking $\beta=\kappa \neq 90^{\circ}$ will reduce the maximum flux, and $\xi \neq \theta(1)$ will change the portion of the time when the hotspot is not visible.

Figure 2 shows the observed flux from the hotspot as a function of the star's precessional and rotational phase. Only half of a precessional period is depicted. The flux varies at twice the precession rate. As Figure 3 shows, the mean flux over the rotational period varies nearly

sinusoidally. Twice during the precessional period, when the mean flux reaches its maximum, the flux does not vary with the rotational phase of the star. At other stages of the star's precession, the hotspot spends much of the rotational period hidden behind the horizon on the neutron star surface. If $\beta=\kappa \neq 90^{\circ}$, one finds that the mean flux varies at the precessional frequency, resulting in a light curve similar to that presented in Figure 4 .

\section{2. $\xi=\kappa=\theta(1)$}

In this case, we attempt to maximize the flux during the portion of the precession when the flux does not vary over the rotation. To do this, we take $\beta=\xi=\kappa=\theta(1)$ and $\gamma=0$. If the bending of the photon trajectories is neglected (i.e. as $y$ approaches zero), $\theta(1)$ approaches $90^{\circ}$ and this case reduces to the previous one. However, for a realistic neutron star with $y \approx 0.2$, we have new light curve which varies at the precession rate (not twice that rate as the previous case). Taking $\beta \neq \kappa$ or $\gamma \neq 0$ also yields a portion of the light curve when the flux does not vary with rotational phase, but this does not coincide with the period when the mean flux reachs its maximum. 

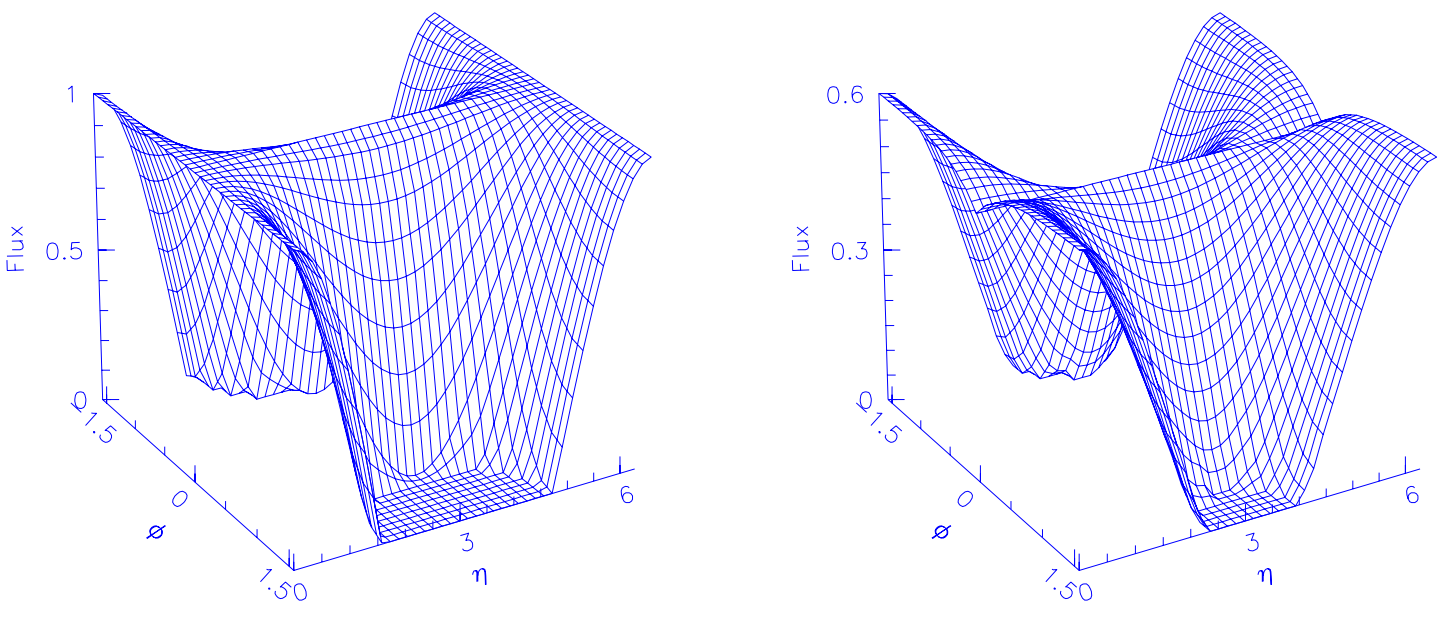

Fig. 2. - Light curves as a function of precessional phase $(\phi)$ and rotational phase $(\eta)$ for $y=0,0.2$ or $R=\infty$ and $11 \mathrm{~km}$ for a $1.4 \mathrm{M}_{\odot}$ neutron star. For both models, $\kappa=\beta=90^{\circ}, \gamma=0$ and $\xi=\theta(1)$. A hotspot on a Newtonian star directly below the observer has a flux of unity in these units.
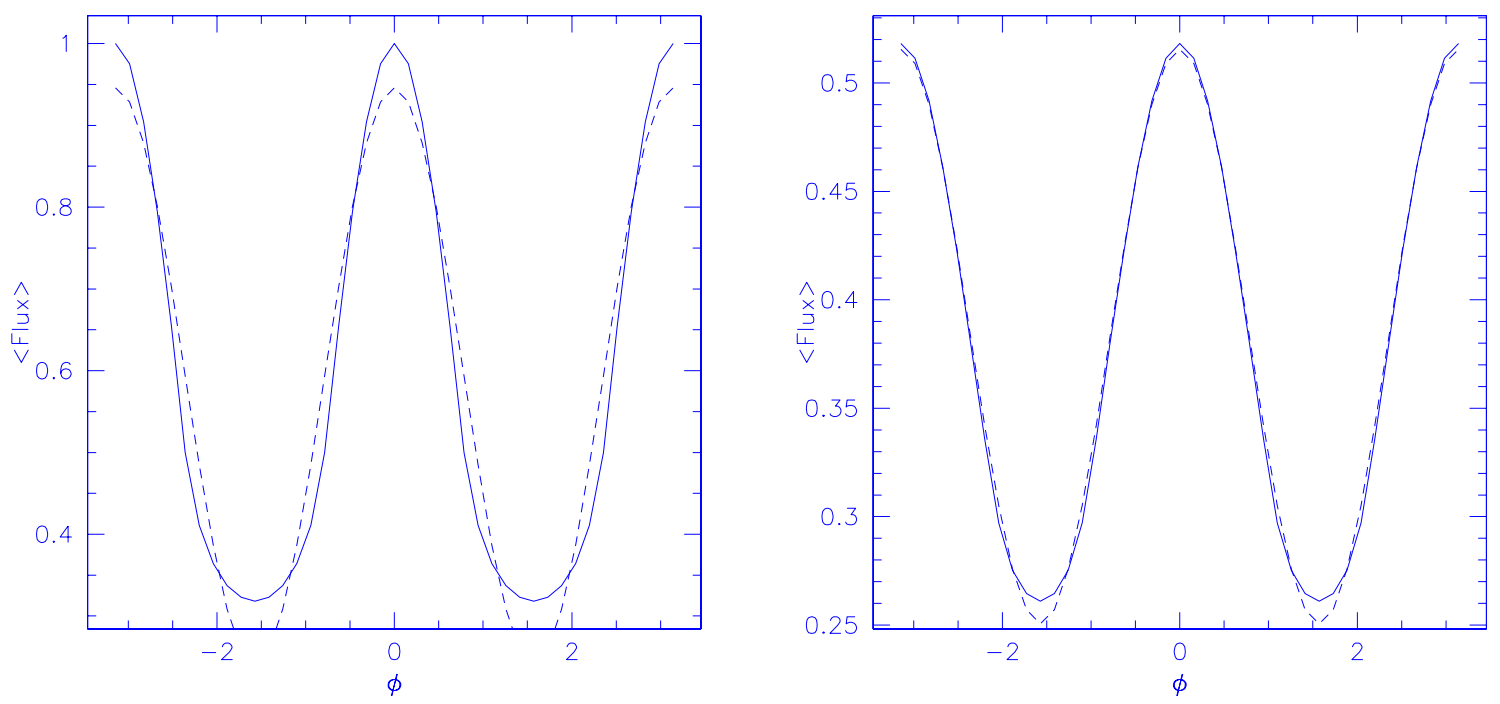

Fig. 3.- Mean flux as a function of precessional phase $(\phi)$ for $y=0,0.2$ or $R=\infty$ and $11 \mathrm{~km}$ for a $1.4 \mathrm{M}_{\odot}$ neutron star. The dashed curve gives the best-fitting function of the form $a \cos 2 \phi+b$. The geometry is as above. 


\subsection{Random Geometry}

The choices of $\xi, \gamma, \beta$ and $\kappa$ that we have made previously are not generic. One would expect the values of $\xi$ and $\gamma$ from a particular neutron star to be random. The values of $\beta$ and $\kappa$ are intrinsic to the star; therefore, one may find a physical motivation for a particular distribution of their values. Figure 5 presents two light curves for two randomly selected geometries.

We created a random sample of several hundred geometries and found that approximately three percent yielded light curves qualitatively similar to those presented in Figure 2 and Figure 4 . Although one would generally expect more complicated light curves such as those depicted in Figure 5, a significant fraction of the geometries yield light curves in which the flux is constant with rotational phase when it reaches its maximum, and during the rest of the precessional period, the hotspot is hidden for a large portion of each rotation.

\section{Discussion}

We have explored several possible light curves of a freely precessing neutron star with a hotspot, and focussed on those whose flux is constant with rotational phase when the flux reaches a maximum value. These possibilities indicate that $1 \mathrm{E}$ 161348-5055 may be a freely precessing neutron star. However, as the mean flux decreases from its maximum it also begins to vary with the rotation of the star; therefore, in the context of this model, we would expect that subsequent observations of 1E 161348-5055 may uncover its rotational period which we would expect to be on the order of several seconds. The variation of the flux with the rotational phase of the star during some portion of the precession appears generic to freely precessing stars with a hotspot.

The Earth undergoes a free precession with a period of about 433 days, known as the Chandler (1891) wobble. This is significantly longer than one would expect from the asymmetry of the Earth's figure due to dissipation inside the Earth (Burs̆a \& Pĕc̆ 1993). Without excitation the wobble would disappear within about a century, and the avenue for its excitation is still unclear.

In neutron stars, precession has been proposed to explain long-term variations in their spin and pulse profiles (e.g. Davis \& Goldstein 1970, Goldreich 1970, Ruderman 1970, Brecher 1972, Pines \& Shaham 1972, Pines, Pethick \& Lamb 1973, Pines \& Shaham 1974). If neutron stars rotate as rigid bodies the precessional period would be $P / \epsilon$ (Pines \& Shaham 1972, Pines \& Shaham 1974) where $P$ is the rotational period. Ruderman \& Sutherland (1974) proposed that neutron stars contain a superfluid component in their cores.

Shaham (1977) explored how the pinning of the superfluid vortices affects the free precession of a neutron star. He argued that the dissipation timescale for the precessional mode $\left(\tau_{w}\right)$ is of the order of the postglitch relaxation time $(\tau)$ times the ratio of the rotational to the precessional frequency. $\tau$ ranges from a week for the Crab to nearly a century for 1641-45 (Shapiro \&

Teukolsky 1983); therefore, depending on the nature of the superfluid coupling the precessional 

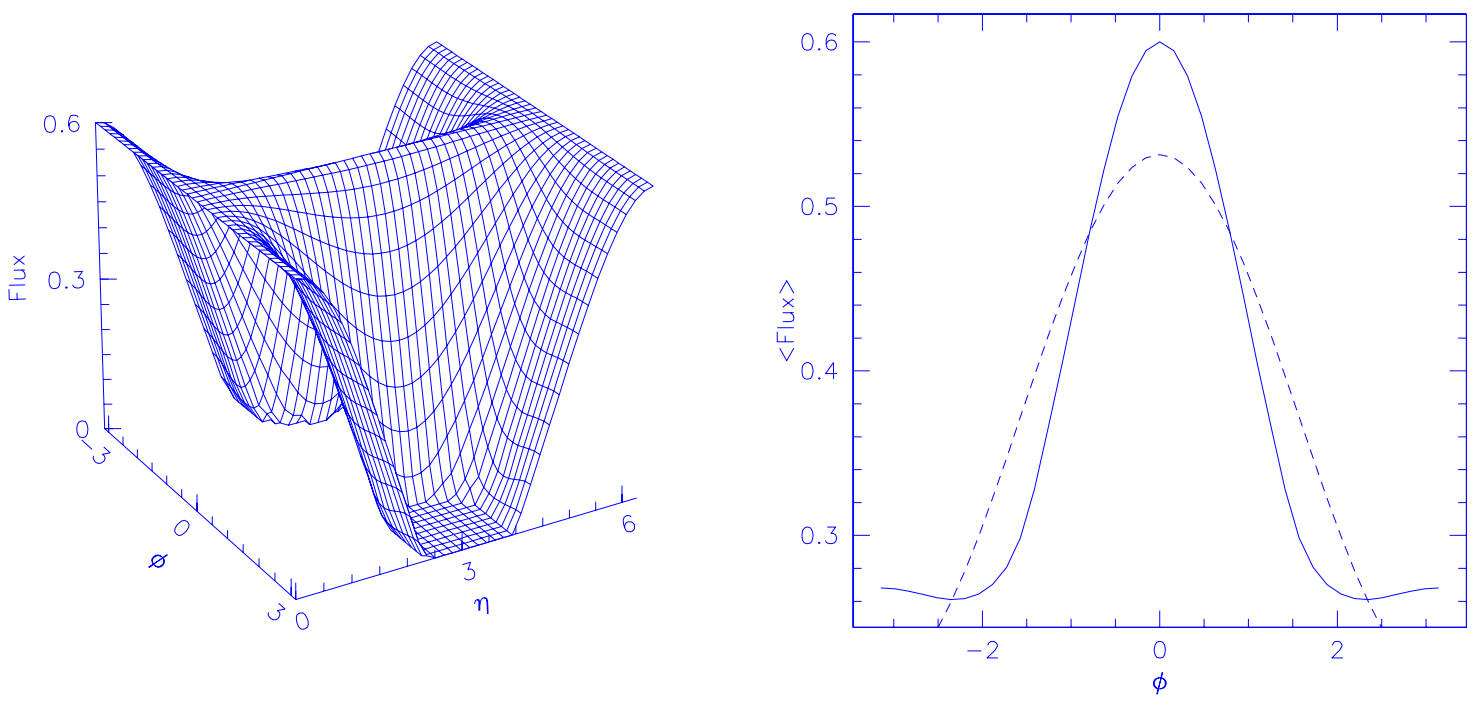

Fig. 4.- The left panel gives the light curves as a function of precessional phase $(\phi)$ and rotational phase $(\eta)$ for $y=0.2 R=11 \mathrm{~km}$ for a $1.4 \mathrm{M}_{\odot}$ neutron star. $\kappa=\beta=\xi=\theta(1)$ and $\gamma=0$ and $\xi=\theta(1)$. The right panel depict the mean flux as a function of $\phi$. The dashed curve gives the best-fitting function of the form $a \cos \phi+b$.
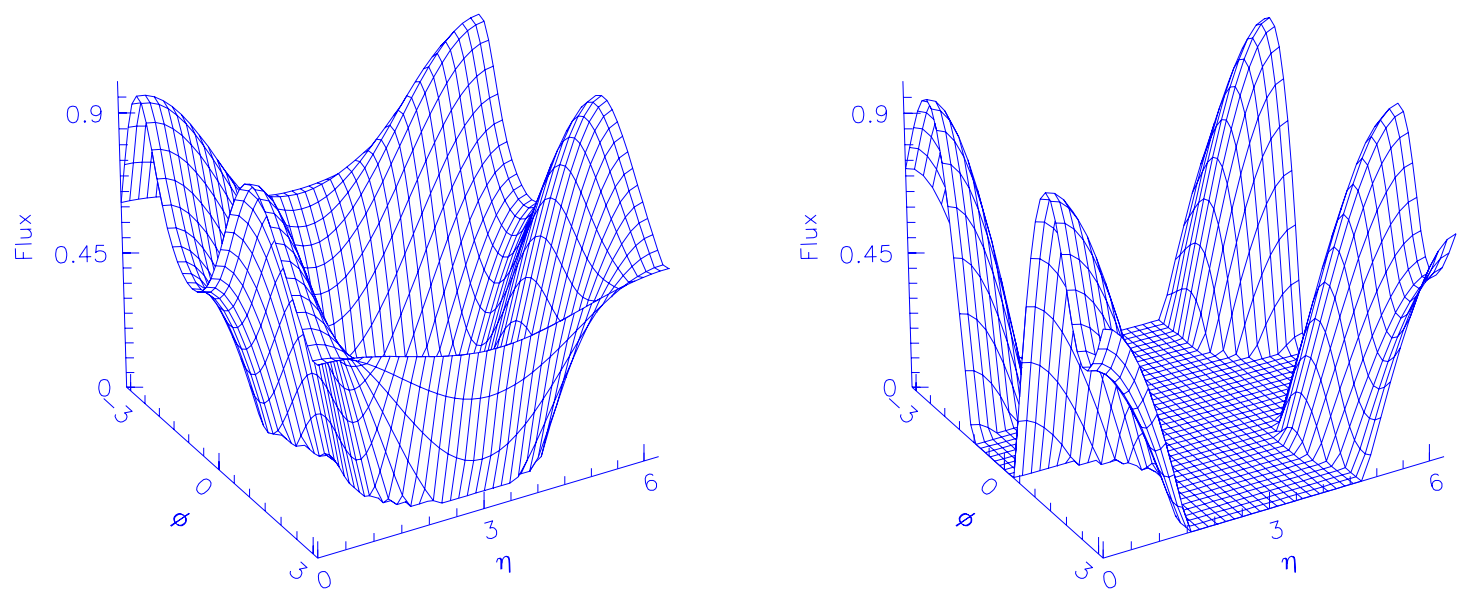

Fig. 5. - The left panel gives the light curves as a function of precessional phase $(\phi)$ and rotational phase $(\eta)$ for $y=0$. The left panel has $\gamma=69^{\circ}, \kappa=144^{\circ}, \beta=62^{\circ}$ and $\xi=115^{\circ}$ and the right panel has $\gamma=138^{\circ}, \kappa=96^{\circ}, \beta=126^{\circ}$ and $\xi=141^{\circ}$. 
mode may last for millennia. Shaham (1977) also found that if the star is triaxial the geometry of the precession is more complicated that for the purely free precession considered here. Additionally, the precessional frequency in this case is given by angular velocity of the superfluid component of the star times the fractional contribution of the superfluid to the total moment of inertia of the star (about one percent).

Sedrakian, Wasserman \& Cordes (1999) have recently reexamined the precession of multicomponent neutron stars with imperfect vortex pinning and found several possibly long-lasting precessional modes with long periods like the precession described here. The excitation and decay of precessional motions in neutron stars are still uncertain.

Evidence has been found for free precession in some radio pulsars (e.g. Cadez, Galicic \& Calvani 1997, Jones 1988), but it is not generic (e.g. Morgan et al. 1995); therefore, the question arises as to which properties of a neutron star would allow or prevent it from precessing and would they correlate with its radio emission. Melatos (1999) and Melatos (2000) argue that precession is characteristic of strongly magnetized neutron stars (c.f. Equation 2). Usov \& Melrose (1996) and Arons (1998) have proposed that strongly magnetized neutron stars are unlikely to produce radio emission collectively due to the formation of bound electron-positron pairs. Alternatively, Baring $\&$ Harding (1997)] suggest that in sufficiently strong fields $\left(B \gtrsim B_{c}\right)$ the QED process of photon splitting (Adler 1971; Heyl \& Hernquist 1997) can dominate one-photon pair production. This will effectively quench the pair cascade, making coherent pulsed radio emission impossible.

Since the timescales for both the excitation and decay of precessional motion in neutron stars are unknown, one can appeal to the relative youth of $1 \mathrm{E}$ 161348-5055 and the other members of the AXP class. They are all several thousand years old, much younger than vast majority of radio pulsars (Taylor, Manchester \& Lyne 1993). The appropriate timescales for precession may simply be shorter than the ages of most radio pulsars while longer than those of AXPs. Furthermore, the hints of precession seen in the Crab pulsars (Cadez, Galicic \& Calvani 1997) point toward this explanation.

We have examined the light curves of free precessing neutron stars with a hotspot and focussed on those geometries which exhibit an epoch during each precessional period where the flux does not vary as the star rotates. These geometries account for about three percent of a random sample and may provide an explanation for the emission from 1E 161348-5055. If this is the case, further observations of the light curve from 1E 161348-5055 should reveal a pulse period of the order of $10^{-4}$ times the precessional period of six hours. Free precession may be a hallmark of young or highly magnetized neutron stars, and it is a direct probe of the structure of the crust and interior of the neutron star and the coupling between them.

\section{REFERENCES}

Adler, S. L. 1971, Ann. Phys., 67, 599. 
Arons, J. 1998, in S. Shibata \& M. Sata (eds.), Neutron Stars and Pulsars, Universal Academy Press, Tokyo, (astro-ph/9802198)

Baring, M. G. \& Harding, A. K. 1997, in C. D. Dermer, M. S. Strickman \& J. D. Kurfess (eds.), Proceedings of the fourth Compton Symposium : Williamsburg, VA April 1997, No. 410 in AIP conference proceedings, p. 638, AIP Press, New York, astro-ph/9711211

Brecher, K. 1972, Nature, 239, 325.

Brecher, K. 1975, Nature, 257, 203.

Burs̆a, M. \& Pěc̆, K. 1993, Gravity Field and Dynamics of the Earth, Springer, Heidelberg

Cadez, A. \& Galicic, M. 1996, A\&A, 306, 443.

Cadez, A., Galicic, M. \& Calvani, M. 1997, A\&A, 324, 1005.

Chandler, S. C. 1891, AJ, 11, 59.

Davis, L. \& Goldstein, M. 1970, ApJ, 159, L81.

Garmire, G. P., Pavlov, G. G., Garmire, A. B. \& Zavlin, V. E. 2000, IAU Circ., 7350, $2+$.

Goldreich, P. 1970, ApJ, 160, L81.

Goldstein, H. 1980, Classical Mechanics, Addison-Wesley, Reading, Massachusetts, second edition

Heyl, J. S. \& Hernquist, L. 1997, Phys. Rev. D, 55, 2449.

Jones, P. B. 1988, MNRAS, 235, 545.

Melatos, A. 1999, ApJ, 519, L77.

Melatos, A. 2000, MNRAS, 313, 217.

Morgan, J. J., Weisberg, J. M., Despotes, J. T., Everett, J. E. \& Cordes, J. M. 1995, in American Astronomical Society Meeting, Vol. 187, p. 1603

Ostriker, J. P. \& Gunn, J. E. 1969, ApJ, 157, 1395.

Page, D. 1995, ApJ, 442, 273.

Pines, D. \& Alpar, M. A. 1985, Nature, 316, 27.

Pines, D., Pethick, C. J. \& Lamb, F. K. 1973, Ann. NY Acad. Sci., 224, 237.

Pines, D. \& Shaham, J. 1972, Phys. Earth and Planetary Interiors, 6, 103.

Pines, D. \& Shaham, J. 1974, Nature, 248, 483. 
Ruderman, M. A. 1970, Nature Phys. Sci., 225, 838.

Ruderman, M. A. \& Sutherland, P. G. 1974, ApJ, 190, 137.

Sedrakian, A., Wasserman, I. \& Cordes, J. M. 1999, ApJ, 524, 341.

Shaham, J. 1977, ApJ, 214, 251.

Shapiro, S. L. \& Teukolsky, S. A. 1983, Black Holes, White Dwarfs, and Neutron Stars, WileyInterscience, New York

Tananbaum, H., Gursky, H., Kellogg, E. M., Levinson, R., Schreier, E. \& Giacconi, R. 1972, ApJ, 174, L143.

Taylor, J. H., Manchester, R. N. \& Lyne, A. G. 1993, ApJS, 88, 529.

Usov, V. V. \& Melrose, D. B. 1996, ApJ, 464, 306. 